\title{
Preußen und Italien im 19. Jahrhundert
}

\author{
Christoph Helm
}

„Jede Generation und jedes Individuum erlebt eine Zeit der Entscheidung, in der zwischen diesen und jenen Möglichkeiten die Wahl fällt zugunsten einer einzigen, die zur Wirklichkeit zu verdichten fortan alle Kraft eingesetzt wird. Diese Entscheidung fällt bei dem frühentwickelten Friedrich Wilhelm bereits zu Beginn der zwanziger Jahre seines Lebens, und es war die brennende Sehnsucht nach dem Süden, die die Reife herbeiführte. Was er damals wurde, blieb er auch."[1]

Es gehört zu den glücklichen Umständen der ansonsten schwierigen geschichtlichen Entwicklung des preußischen Staatswesens im 19. Jahrhundert, dass das, was hier über den 1795 geborenen Kronprinzen Friedrich Wilhelm, den späteren König Friedrich Wilhelm IV, gesagt wurde, für eine Reihe weiterer Persönlichkeiten jener geschichtlichen Epoche gilt, die bis heute erkennbar gestaltend die Kunst- und Kulturgeschichte Preußens beeinflusst haben, gemeint sind vor allem Karl Friedrich Schinkel und Ludwig Ferdinand Hesse. Allen dreien ist gemeinsam, dass sie sich in Kongenialität zunächst der prägenden Kraft der romantischen Ideenwelt und dann der Klassik hingaben und dass für sie die überzeugenden Vorbilder der profanen und kirchlichen Bauten vorrangig in den Architekturleistungen des Südens aus Antike und Mittelalter bestanden. Für jeden der drei gilt weiterhin, dass der lang herbeigesehnte und schließlich erwirkte Aufenthalt in Italien zu einer dauerhaften Verstärkung der geistesgeschichtlichen Grunddispositionen führte, die mit organisch sich zusammenfügenden Neuanregungen verbunden waren.

Wahr ist aber auch, dass diese glückhaften Umstände, die mit den Lebensprägungen von Individuen zusammenhängen, sich in die ideengeschichtlichen Strömungen einbetteten, die seit dem Tode Friedrichs des Großen das Königreich Preußen bewegten. Es ist dem von der Geschichte und der Geschichtsforschung verkannten und stiefmütterlich behandelten Nachfolger des großen Königs zu verdanken, dass es nach 1786 in Architektur und Kunstgeschichte zu deutlichen Neuansätzen kam, die zur Befreiung aus der zur Statik und Erstarrung hinneigenden Spätphase des Barock und Rokoko führten.[2] In den nur elf Jahren seiner Regierungszeit hat Friedrich Wilhelm II durch Reform der Architektenausbildung und Berufung der Baumeister Friedrich Wilhelm v. Edmannsdorf, Carl Gotthard Langhans und Carl v. Gontard sowie des Bildhauers Johann Gottfried Schadow wesentliche Voraussetzungen zur baulichen Weiterentwicklung und Umgestaltung seiner Residenzen Berlin und Potsdam geschaffen, die über Preußen hinaus Auswirkungen auf das Heilige Reich und Europa insgesamt hatten.[3] In der Windstille, die abgesetzt von der von Frankreich ausgehenden revolutionären Dynamik jenseits der Elbe noch herrschte, entfaltete Preußen Wirkung vorrangig durch seine kulturelle Entwicklung, die hauptsächlich durch die von Langhans und seiner Schule initiierte klassizistische Bauweise, die sich am eindrucksvollsten im Brandenburger Tor (1789), der Orangerie im Neuen Garten (1791) sowie im Marmorpalais Friedrich Wilhelms II (1787 - 92) manifestierte, stilbildend auf Europa wirkte. In den Jahren scheinbarer Ruhe vor dem Sturm über Europa, den Napoleon entfachte, bereiteten sich im Preußen Friedrich Wilhelms II sichtbar die Kräfte in Kunst, Kultur und Wissenschaft vor, die nach der Demütigung von Jena und Auerstädt den Selbstbehauptungswillen des preußischen Staates verkörperten und nach 1810 dem Neubeginn durch staatliches Handeln erkennbar Ausdruck verliehen. Welche Kräfte hier gleichsam nach einer Inkubationszeit zum Aufbruch kamen, zeigt sich paradigmatisch an den noch in der Spätzeit Friedrich Wilhelms II initiierten Planungen für ein Berliner Monumentum für Friedrich den Großen. Die sich an der klassischen Antike orientierenden Ideen des Wettbewerbes für das Monumentum, die ausgehend von Langhans und Friedrich Gilly schon Karl Friedrich Schinkel spürbar beeinflussten, haben während des gesamten 19. Jahrhunderts Auswirkungen auf die Bauplanungen in Berlin und Potsdam gehabt, um schließlich in der zweiten Hälfte des 19. Jahrhunderts in der Gestaltung des Schauspielhauses und der Nationalgalerie ihre konkretisierte Form der Realisierung zu finden.

Eine Darstellung der Beziehungen Preußens und Italiens im 19. Jahrhundert muss beschreibend darüber hinaus auf den Kreis Bezug nehmen, der sich seit 1800 in Rom um den Botschafter Preußens am Heiligen Stuhl, Wilhelm v. Humboldt, und dessen Gattin Caroline gebildet hatte. Die hier an der Via Gregoriana Versammelten, die Familie des Botschafters und ihr Freundeskreis, der aus Schriftstellern, Malern, Architekten, Musikern und Bildhauern aus dem Norden bestand, sie waren gleichsam imprägniert von dem Geist und der Anmut Roms und wünschten, einen möglichst großen Teil der in Italien gewonnenen Inspirationen in eigenes verantwortliches Handeln umsetzen zu können. Zu diesen Künstlern am Monte Trinità gehörten unter anderem die Maler Christian Reinhart, Joseph Anton Koch und Gottlieb Schick, die Dichterin Friederike Brun und der Bildhauer Berthel Thorwaldsen, die sich beinahe täglich in der Residenz des Botschafters oder in der nahebei gelegenen römischen Taverne, die sich „Griechisches Café" nannte, trafen und ihre Ideen austauschten. Ein gelegentlicher Gast war auch Alexander v. Humboldt, der Bruder des Botschafters, der diesen Kreis durch seine Berichte über seine naturkundlichen Forschungsreisen bereicherte.

Sie alle charakterisierte, dass sie in Rom durch die lebendige Anschauung der Altertümer einen vorzüglichen Eindruck von der Antike erhielten, soweit damals überhaupt schon Gesamteindrücke möglich waren[4], und dass dies prägend wurde für ihr Lebenswerk. Darüber hinaus ist bemerkenswert, dass in dieser verdichteten Sphäre der 
Inspiration in Rom im Jahre 1804 mit Wilhelm v. Humboldt und Karl Friedrich Schinkel zwei Persönlichkeiten zueinander fanden und eine lebenslange Freundschaft begründeten, die auf den verschwisterten Gebieten der Wissenschaft sowie Kunst und Architektur jeweils einzigartig werden sollten und sich zu Schlüsselfiguren ihrer Fachrichtung entwickelten. Es ist hier nicht die Zeit, auf die Reformen Humboldts in Bildung und Wissenschaft für Preußen und Deutschland nach 1810 im Detail einzugehen.[5] Nur soviel sei gesagt. Die Grundstrukturen des Bildungs- und Wissenschaftssystems, die von einem organischen Gefüge und Aufbau von Schule und Universität ausgehen und die Voraussetzungen bildeten für die einzigartige Weltgeltung, die Bildung und Wissenschaft in Preußen und Deutschland bis 1918 besaßen, sind ohne die Idee des Humanismus und das überzeugende Bildungsideal der Wertvorstellungen der Antike nicht denkbar. In der Stringenz der Ausprägung dieses bildungs- und wissenschaftspolitischen Ideals, das wir Neuhumanismus zu nennen uns angewöhnt haben, spiegeln sich die unmittelbaren und ungetrübten Eindrücke wider, die Humboldt in seiner Zeit in Rom aufgenommen hatte und die ihm die innere Kraft für sein großartiges Reformwerk vermittelten. Gleichzeitig hatte Humboldt nach 1810 durch sein Amt in Berlin die Möglichkeit, für Schinkel einen großzügigen Handlungsrahmen zu setzen, der dessen Fähigkeiten adäquat war. Was in Rom 1804 mit der Grabstele für den früh verstorbenen Sohn Humboldts, Wilhelm, begann, nahm seine einzigartige Fortsetzung nach den Befreiungskriegen gegen Napoleon in der kreativen Schaffensfreude Schinkels in Preußen.

Schinkel wurde der Baumeister Preußens in der Phase der beginnenden Industrialisierung, zu einer Zeit also, als sich die Bevölkerungszahl Berlins zwischen 1815 und 1841 beinahe verdoppelte. Die darin liegenden Möglichkeiten wurden allerdings begrenzt durch den Sparwillen, der Friedrich Wilhelm III auszeichnete, der sich exemplarisch auch darin manifestierte, auf den Bau eines eigenen Schlosses zu verzichten. Trotzdem fand Schinkel natürlich vorzügliche Rahmenbedingungen vor, zumal er mit Peter Joseph Lenné einen Gartenarchitekten hatte, mit dem ihn kongeniale Auffassungen verbanden. Ohne durch etwaige Stilvorschriften des Königs eingeengt zu werden, konnten Schinkel und Lenné gemeinsam daran gehen, eigene Vorstellungen baulich zu realisieren, so dass sich Berlin vor allem durch die Bauten im Lustgarten und auf dem Gendarmenmarkt, die durch ihr klassisches Gepräge das Erscheinungsbild der wachsenden Großstadt nachhaltig veränderten, den Ruf als Spree-Athen erwarb, der gleichzeitig Ausdruck der nach 1830 aufgrund des Freiheitskampfes der Hellenen einsetzenden Griechenlandbegeisterung der Berliner war.[6] Durch Schinkel erhielten Berlin und Potsdam Grundstrukturen, die bis heute nachwirken. Es entbehrt hierbei nicht einer gewissen Tragik, dass die großen Möglichkeiten, die für Schinkel in der Thronbesteigung Friedrich Wilhelms IV 1840 lagen, mit dem ihn trotz gewisser Nuancen in ihren kunsthistorischen und stilistischen Präferenzen eine innige Freundschaft verband, nicht mehr zur Umsetzung gelangen konnten. So bleibt nur reizvolle Spekulation, wie der romantische Baukünstler und der visionäre Architekt durch Kreativität der Zusammenarbeit
Realitäten geschaffen hätten, wenn nicht Schinkels Tod 1841 diese Möglichkeit abrupt beendet hätte.

Noch von einer weiteren Konstellation soll hier berichtet werden, die über den engen Rahmen ihres Genres, der Malerei, hinaus Wirkung entfaltete durch die Intensität der Inspiration, die Deutsche in Rom empfingen, gemeint ist die Malergruppe der Lukasbrüder oder Nazarener, die zu Beginn des 19. Jahrhunderts in Rom bestand. Von frühromantischen Vorstellungen geprägt waren sie abgestoßen von der Entwicklung, die die französische Revolution mit Jakobinertum, Direktorium und Kaisertum Napoleons eingeschlagen hatte. Hinzu trat eine Abneigung gegen die bürgerliche Welt mit ihren als bedrohlich empfundenen Vereinnahmungstendenzen sowie gegen die herrschenden Strömungen in den Kunstakademien in Deutschland, die keinen Raum für neue Ideen zuzulassen schienen. Dies alles führte zu einer bohèmienhaften Absonderung in der römischen Diaspora, die in Form eines durch Freundschaft charakterisierten Männerbundes, der mittelalterliche Riten aufleben ließ, praktiziert wurde und in dem Gefühl erfolgte, einer Avantgarde anzugehören.

Das Idealbild dieser Gruppe war die Kunst und die Lebensform des Mittelalters, wobei diese nach ihrer Überzeugung durch die unitas imperii et fidei, die Einheit des Abendlandes unter kaiserlicher Schutzherrschaft und behütet durch den katholischen Glauben, jene durch hohe handwerkliche Präzision und eingebettet in die Vorstellungswelt der Mutter Kirche charakterisiert war. Hieraus ergab sich einer Verehrung altdeutscher Malerei, die Wertschätzung handwerklicher Kunst sowie eine Vorliebe für religiöse Motive. Einige der Lukasbrüder konvertierten zum katholischen Glauben, weil dieser ihrem Lebensentwurf entgegenkam und als Familienersatz Schutz zu bieten schien. Die Rückwirkungen dieser Deutsch-Römer auf Preußen waren vielfältiger Natur. So ergab sich aus der Vorliebe mittelalterlicher Kunst ein Wiederentdecken künstlerisch-handwerklicher Fertigkeiten und Techniken bis hin zur Präferenz bestimmter Farbkompositionen, was sich befruchtend auf die Kunstentwicklung in Deutschland auswirkte.

Am sinnfälligsten ist dies bei der Ästimierung der Fresko-Malerei durch die Nazarener, die sich auf historische Vorbilder in Antike und Mittelalter beziehen konnte. Es war der Generalkonsul Preußens in Rom, Jacob Salomon Bartholdy, der 1816 die Bedeutung der Lukas-Bruderschaft erkannte und ihr den Auftrag erteilte, Teile der Casa Zuccari an der Piazza della Trinità dei Monti in Fresko-Technik auszumalen.[7] Der Auftrag ging an die preußischen Staatsbürger Philipp Veit, Peter Cornelius, Wilhelm Schadow, den Sohn Johann Gottfried Schadows, [8] sowie an den aus Lübeck stammenden Friedrich Overbeck, der als echtes Gemeinschaftswerk der Gruppe in nicht ganz einem Jahr erfüllt werden konnte. Die Fresko-Malerei in der Villa Bartholdy, inhaltlich der alttestamentarischen Josephslegende gewidmet, bedeutete für die Lukas-Brüder die erfolgreich bestandene Herausforderung, technisch und stilistisch an die italienische Renaissance anzuknüpfen und diese in Deutschland zu propagieren.

Die Schlüsselfigur hierzu wurde Wilhelm Schadow, der 1819 aus Rom nach Preußen zurückgekehrt war und als einer der Begründer der Monumentalmalerei bezeichnet werden kann. Durch ihn und den Einfluss der nazarenisch 
geprägten Stilrichtung, die nach 1820 vorherrschend wurde, traten nun zunehmend Bilder mit religiösen Inhalten und solchen, die sich an altdeutschen, niederländischen oder italienischen Motiven orientierten, in den Vordergrund. Gleichzeitig nahm die Monumentalmalerei erstmals auch durch staatliche Aufträge, die König Friedrich Wilhelm III auslöste, einen enormen Aufschwung. Den Anfang machte sein Auftrag, eine Abfolge von Gemälden für die Garnisonskirche in Potsdam anzufertigen, es folgte die Ausmalung des Berliner Schauspielhauses sowie der Vorhalle und des Treppenhauses im Alten Museum. Es ist bemerkenswert, dass die Entwürfe für die Werke im Alten Museum von Karl Friedrich Schinkel stammen und sich somit interessante Querverbindungen zu den „DeutschRömern“ herstellen lassen, die vor den Nazarenern entscheidende Prägungen in Italien empfangen hatten.

Der Aufschwung der Monumentalmalerei, die in einer ihrer Stilrichtungen preußisch-patriotischen Charakter entwickelte, hing mit einer weiteren Veränderung zusammen, die sich daraus ergab, dass infolge der Regelungen des Wiener Kongresses das Rheinland an Preußen fiel mit dem Auftrag, die Wacht am Rhein zu übernehmen. Dies verstärkte zum einen den patriotischen Zug der Monumentalmalerei, gab aber andererseits den bisher im protestantischen Norden und Osten tätigen nazarenisch ausgerichteten Künstlern um Wilhelm Schadow eine verbreiterte Plattform, die ihren Möglichkeiten noch besser gerecht wurde und zusätzliche Anregungen erlaubte. 1826 wurde Wilhelm Schadow zum Direktor der Düsseldorfer Akademie berufen, die unter seiner Ägide rasch zu internationalem Ruhm aufstieg und sich zu einer gewichtigen nazarenisch geprägten Schule entwickelte, die sich als eigenständiges Kraftzentrum darstellte und durch ihre ungebundene Kreativität Berlin zu überflügeln schien. Überhaupt ergab sich die eigentümliche Situation, dass die Nazarener, als „verirrte Enkel“ Johann Joachim Winckelmanns[9] ursprünglich dezidierte Gegner der bürgerlich geprägten deutschen Akademietraditionen, nun daran gingen, die Führungspositionen in diesen Akademien zu übernehmen. So wurde Peter Cornelius Direktor der Akademie in München, Julius Schnorr v. Carolsfeld Direktor der Galerie in Dresden und Philipp Veit wirkte zunächst als Direktor des Städels in Frankfurt/Main und danach der Galerie in Mainz. Vor diesem Gesamthintergrund und dem zunehmenden Einfluss der Düsseldorfer Schule in den dreißiger Jahren des 19. Jahrhunderts wird es verständlich, dass einerseits Freskenzyklen und Historienmalerei als zentrale künstlerische Impulse angesehen wurden, andererseits auch die Landschaftsmalerei anfangs von dieser Stilrichtung beeinflusst war.

Zur selbständigen Gattung erhoben, ohne die Herkunft aus Romantik und Klassik zu leugnen, hat die Landschaftsmalerei indessen erst das Wirken von Carl Blechen. Er wurde der Landschaftsmaler Preußens par excellence, dem 1828 mit dem Gemälde Semnonenlager auch international der Durchbruch gelang. Erstmals ist hier in Verbindung von mythologischer Fiktion - der germanische Stamm der Semnonen wird als Träger des Widerstandes gegen die Römer dargestellt - mit heimischen Landschaftsbezug - die Szene ist in den Müggelbergen bei Berlin zu lokalisieren - eine Konkretisierung der sagenhaften märkischen Vorge- schichte vorgenommen worden, die beispielgebend wurde für die weitere Entwicklung der Historienmalerei. Aufbauend auf diesen künstlerischen Ansätzen gewann Blechen weitere wesentliche Anregungen durch seine Italienreise 1828/29, die faszinierend für ihn wurde durch das Erlebnis der Helligkeit und der Farben des Südens, durch deren Expression von nun an seine Werke charakterisiert sind. Dies zeigt sich wesentlich in den insgesamt fünf Gemälden mit Innenansichten des Palmenhauses auf der Pfaueninsel, die nach 1834 im Auftrage König Friedrich Wilhelms III entstanden und als frühe Beispiele der Architekturmalerei gelten können. So wurde auch für Carl Blechen das Italienerlebnis wesensbestimmend und die Herausforderung der Wiedergabe der südländischen Lichteffekte hat sein weiteres Lebenswerk beherrscht.

Ebenfalls im Jahr 1828 konnte Kronprinz Friedrich Wilhelm mit Erlaubnis seines königlichen Vaters seine Italienreise antreten, die allerdings auf nur zwei Monate begrenzt war, so dass das Besuchsprogramm einen eklektischen Charakter erhielt. Liest man indes die enthusiastischen Briefe, die Friedrich Wilhelm seiner Gattin Elisabeth von dieser Reise sandte, erhält man einen Eindruck von der Begeisterungsfähigkeit des Prinzen, dessen anima, wie in Platos Ideenlehre beschrieben, nun das Urbild der Wesensdinge im Original endlich wiederzufinden schien, die immer in ihm waren[10]. Die Überfülle der Eindrücke, die Überfülle der Wiederentdeckungen verdichteten sich bei ihm in besonderer Weise beim Anblick Roms, Pompejis und Venedigs, die reiche Ideenskizzen für kirchliche und profane Bauten lieferten, wobei sich eine gewisse Präferenz für antike Villen- und Hausanlagen bemerkbar machte. Dies ging bei ihm in kunsthistorischer Hinsicht bezüglich der Profanbauten Hand in Hand mit einem leichten Übergang vom Klassizismus hin zur italienischen Hochrenaissance, die er von nun an als Stilrichtung bevorzugte, während bei den Sakralbauten eine Vorliebe für Grundrisse frühchristlicher Basiliken erkennbar wurde, die der Prinz unter anderem bei der Besichtigung des Forum Romanum kennen gelernt hatte. Es ist hier nicht die Zeit, auf alle Facetten und Einzelheiten der Italienreise Friedrich Wilhelms einzugehen. In der Summe bedeutete aber die Akzentverschiebung seiner kunsthistorischen und stilistischen Präferenzen nach 1828 bei aller Vertrautheit der persönlichen Beziehungen auch eine gewisse Distanz zu dem eine halbe Generation älteren Schinkel, der der klassizistischen Stilrichtung verpflichtet blieb.

Unter so veränderten Rahmenbedingungen erhielt nach der Thronbesteigung Friedrich Wilhelms 1840 und dem Ableben Schinkels 1841 eine neue Generation von Baumeistern, die allerdings alle Schüler von Karl Friedrich Schinkel waren, ihre Bewährungschancen, nämlich Ludwig Persius, August Stüler und Ludwig Ferdinand Hesse. Während Persius als Architekt für Potsdam und Stüler als solcher für Berlin Verantwortung trugen, übernahm nach beider Tod Hesse jeweils deren Funktion. In der Tradition Schinkels stehend trug Hesse somit Verantwortung in Potsdam und Berlin.

Mit Friedrich Wilhelm IV verband Hesse die tiefe Liebe und Verehrung Italiens, die auch Ergebnis einer ausgedehnten Reise waren, die Hesse 1834 nach Italien unternehmen konnte. Florenz, Rom, Neapel und Sizilien waren 
die Stationen dieser Bildungsreise, die weitestgehend frei von zeitlichem Druck gestaltet werden konnte. Darüber hinaus war Hesse altersgleich mit dem König, was den Umgang miteinander erleichtern konnte. Dieser gewissen vertrauten Situation mit dem König und der königlichen Familie, die auch damit zusammenhing, dass Ludwig Ferdinand Hesse als Hof-Bauinspektor seit 1832 schon für dessen Vater, Friedrich Wilhelm III, tätig gewesen war, ist es vermutlich zu verdanken, dass die enge Zusammenarbeit nach 1861 auch unter dem Nachfolger, dem König und Kaiser (seit 1871) Wilhelm I, jüngerer Bruder Friedrich Wilhelms IV, weitergeführt werden konnte. In Fortsetzung der Aufgaben und des Erbes von Schinkel, Persius und Stüler hat Hesse somit unter drei Königen von Preußen sein Aufgabengebiet gefunden. Von der Kontinuität, die hierbei in der Baukunst Preußens im 19. Jahrhundert gefunden wurde, die sich durch eine tief empfundene Verbundenheit zur klassischen Antike und Hochrenaissance Italiens auszeichnet, zeugen bis heute die Baudenkmale dieser Zeit in Potsdam und Berlin. Bezüglich Ludwig Ferdinand Hesses sind hierbei unter anderem neben der Friedenskirche, dem Mausoleum in Charlottenburg und dem Seitenflügel des Marmorpalais vorrangig das Lustschloss auf dem Pfingstberg, die neue Orangerie und das Elisabeth-Krankenhaus zu nennen. Daneben zeugen eine Fülle von Privatbauten in Potsdam und Berlin vom Selbstverständnis dieses Baumeisters, der ein bedeutendes Kapitel preußisch-deutscher Architektur mitgestaltet hat.

\section{Anmerkungen}

[1] Ludwig Dehio, Friedrich Wilhelm IV. von Preußen. Ein Baukünstler der Romantik. Hrsg. von Hans-Herbert Möller, Berlin 2001, S. 23.

[2] Zur Frage historischer Größe und der Bewertung Friedrich Wilhelms II vgl. die klugen und ausgewogenen Bemerkungen von David E. Barcley, Friedrich Wilhelm II, in: Preussens Herrscher. Von den ersten Hohenzollern bis Wilhelm II. Hrsg. von Frank-Lothar Kroll, München 2000, S. 179 ff.

[3] Vgl. Heinz Schönemann, Architektur und Stadtgestaltung, in: Preußen. Kunst und Architektur. Hrsg. von Gert Streidt, Köln 1999, S. 275 ff.

[4] Durch die türkische Besetzung von Hellas war der Zugang zu den griechischen Originalstätten versperrt. Dies änderte sich erst, als mit der Befreiung Griechenlands 1832 erste archäologische Forschungen möglich wurden; vgl. dazu Christoph Helm, Aspekte orientwissenschaftlicher Forschung in Halle. Festvortrag anlässlich der Gründung des orientwissenschaftlichen Zentrums an der Martin-Luther-Universität Halle-Wittenberg, Orientwissenschaftliche Hefte, 1/2001, Halle 2001, S. 1-13.

[5] Vgl. dazu Christoph Helm, Historische Prozesse der Hochschultransformation in Folge der Wiedervereinigung 1990, in: Wissenschaftliche Beiträge der Fachhochschule Lausitz, II. Ausgabe, Cottbus 2003, S. 16-30. Vgl. darüber hinaus ders., Zehn Jahre Wissenschaftsentwicklung in den neuen Bundesländern an Hand des Paradigmas Sachsen-Anhalt. Kurzinformation HIS, Hannover 2001, sowie ders., Hochschulen und Forschungseinrichtungen Ostdeutschlands in Kooperation mit Osteuropa, in: Forum der Forschung. Wissenschaftsmagazin der Brandenburgischen Technischen Universität Cottbus, Nr. 12, Cottbus 2001, S. 13-33.
[6] Aufschlussreich ist in diesem Zusammenhang, dass sich Kronprinz Friedrich Wilhelm in dieser Zeit mit Bauplänen befasste, das Schloss für den ersten griechischen König der Neuzeit, den Wittelsbacher Otto, auf der Akropolis in Athen zu errichten; vgl. Helm, Aspekte, S. 5 f.

[7] Vgl. Manfred Janslin, Die gescheiterte Kulturrevolution. Perspektiven religiös-romantischer Kunstbewegung vor der Folie der Avantgarde. München 1988, S. 70 ff.

[8] Schon dieser hatte sich zu Studienzwecken zwischen 1785 - 1787 in Rom aufgehalten und dort einen Wettbewerb an der Academia di San Luca gewonnen; vgl. Claudia Czoll, Schadow, Sokrates und das Judentum, Berlin 2002, S. 7.

[9] Vgl. Janslin, Kulturrevolution, S. 105 ff.

[10] Vgl. Dehio, Friedrich Wilhelm IV, S. 59 ff.

\section{Autor}

Dr. phil. Christoph Helm

Hermann-Korb-Straße 60

38302 Wolfenbüttel

Tel. +495331 78929

helm@curt-mast.de 